\title{
Tratamiento multidisciplinar del leimiosarcoma de vena cava inferior
}

\author{
Ana Moreno H. ${ }^{1}$, Luz Fernández V. ${ }^{1}$, José Ángel López B. ${ }^{1}$, María Dolores Pérez D. ${ }^{1}$
}

${ }^{1}$ Hospital General Universitario Gregorio Marañón, Madrid España.

Recibido el 17 de enero de 2019 y aceptado para publicación el 19 de marzo de 2019.

Correspondencia a: Dra. María Dolores Pérez D. lolaperezdiaz@hotmail.com

\section{Multidisciplinary treatment of leiomyosarcoma of inferior cava}

Introduction: Leiomyosarcomas of the inferior vena cava are rare tumors, with fewer than 300 cases reported. Its incidence is higher in females, usually appear in the sixth decade and they have a slow-growing and poor prognosis. Symptoms are generally non-specific. Diagnosis is made with imaging studies and guided biopsy. Clinical Case: We report a case of a 73-year-old male patient with leimyosarcoma of the inferior vena cava treated by neoadjuvant radiotherapy, surgery and intraoperative radiotherapy. Discussion: Surgery is the only treatment that can improve the survival. The role of the adjuvancy and neoadjuvancy is very controversial. Surgical management is determined by the location of the tumour, the relationship with adjacent structures and the presence of collateral veins.

Key words: leiomyosarcoma; inferior vena cava; radiotherapy; surgical resection.

\section{Resumen}

Introducción: Los leiomiosarcomas de la vena cava inferior son tumores raros, sólo hay 300 casos descritos en la literatura. Su incidencia es mayor en mujeres, suelen aparecer entre los 50-60 años, y presentan una progresión lenta y mal pronóstico. Los síntomas son inespecíficos haciendo que el diagnóstico se realice de forma tardía, éste se realiza mediante pruebas de imagen y biopsia guiada. Caso clínico: Se presenta el caso de un varón de 73 años con diagnóstico de leiomiosarcoma de la vena cava inferior, como hallazgo incidental en TC de control, tratado mediante radioterapia neoadyuvante, cirugía y radioterapia intraoperatoria. Discusión: El único tratamiento que ha descrito modificaciones en la supervivencia es la cirugía. El papel de la adyuvancia y neoadyuvancia en estos tumores es muy controvertido. La elección de la actitud terapéutica dependerá de la localización del tumor, tamaño, la relación con estructuras adyacentes y la presencia de circulación colateral.

Palabras clave: leiomiosarcoma; vena cava inferior; radioterapia; resección quirúrgica.

\section{Introducción}

Los tumores malignos de grandes vasos son extremadamente raros. El leimiosarcoma de la cava inferior (VCI) es el más común ${ }^{1}$; desde la primera descripción de Pearl en 1871, se han publicado un total de 300 casos, siendo el $80 \%$ en mujeres entre la $5^{\text {a }}$ y $6^{\text {a }}$ década de la vida ${ }^{2-8}$. Los síntomas son inespecíficos haciendo que el diagnóstico sea tardío en muchos casos, realizándose mediantes pruebas radiológicas y biopsia guiada.

El leiomiosarcoma es un tumor maligno de origen mesenquimal originado a partir de la musculatura lisa de la túnica media. Este tumor tiende a presentar gran tamaño sin causar metástasis y a crecer a lo largo de la luz del vaso. Clásicamente, se asocia a una progresión lenta y mal pronóstico, y el único tratamiento que ha descrito modificaciones en la supervivencia es la cirugía radical, de hecho el principal indicador pronóstico es la resección quirúrgica con márgenes negativos ${ }^{1}$.

Actualmente, la radioterapia (RT) preoperatoria parece que puede producir una disminución del tamaño tumoral y facilitar la cirugía, y la radioterapia intraoperatoria (RIO) puede contribuir a reducir la tasa de recurrencia local y los efectos secundarios de la radioterapia sistémica, pero no hay un consenso en cuanto a la mejor estrategia terapéutica ${ }^{1}$.

Presentamos el caso de un paciente con un leio- 
miosarcoma de VCI en el que se hizo un abordaje multidisciplinar.

\section{Caso clínico}

Se presenta el caso de un varón de 73 años con antecedentes de dislipemia, hipertensión arterial, hernia de hiato y hemocromatosis (heterocigoto H63D) tratada con flebotomías. Durante su seguimiento en consultas de cirugía por un pólipo vesicular se objetiva en una ecografía abdominal una masa sólida retroperitoneal derecha confirmada con TC abdomino-pélvico, que la informa de masa retroperitoneal infrarrenal paracava derecha compatible con liposarcoma retroperitoneal $(63 \times 52 \mathrm{~cm})$, en contacto con la división iliaca arterial derecha, el músculo psoasilíaco derecho y la tercera porción duodenal (Figura 1). El resultado histológico obtenido a partir de una biopsia con aguja gruesa (BAG) (Figura 2) guiada por TC fue de tumor mesenquimal fusocelular, con perfil inmunohistoquímico compatible con leiomiosarcoma con un Ki 67de 20\%.

El caso fue presentado en el Comité de Sarcomas de nuestro centro donde existe un protocolo de RT neoadyuvante y cirugía radical con radioterapia intraoperatoria (RIO).

El paciente recibió una dosis total de 4.500 cGy de RT externa preoperatoria distribuida en un total de 25 sesiones. En el TC de reevaluación no se observaron cambios significativos. Se realizó la intervención quirúrgica observándose una masa retroperitoneal de gran tamaño que englobaba la pared derecha de la VCI desde la vena renal de ese lado hasta la bifurcación de las iliacas y sin infiltración duodenal (Figura 3). Se llevó a cabo la liberación del parietocólico derecho y exposición de la masa mediante la maniobra de Cattell-Braasch, con posterior identificación de uréter derecho, verificando la no infiltración del mismo. Se realizó control vascular proximal y distal, respetando ambas venas renales y seccionando la vena cava con endograpadora, posteriormente se llevó a cabo la exéresis en bloque de la vena cava inferior infrarrenal consiguiendo una resección completa del tumor. Tras la extirpación de la masa tumoral, se administraron 1.250 cGy por campo de forma directa sobre el lecho quirúrgico (Figura 4). Se decidió no realizar reconstrucción vascular por el alto riesgo de trombosis de la prótesis.

El paciente subió a la planta el mismo día de la cirugía y el tercer día posoperatorio presentó dolor en miembro inferior derecho, edema y aumento de temperatura compatible con cuadro de trombosis venosa profunda (TVP) que, posteriormente, fue confirmado por ecodoppler, por lo que se inició tratamiento anticoagulante con heparinas de bajo peso molecular con evolución favorable.

La anatomía patológica informó de leiomiosarcoma G2 de dimensiones 8,5 x 7 x 6,4 cm, y un índice de proliferación Ki $67 \%$ del $30 \%$.

El caso clínico fue presentado de nuevo en el Comité de Sarcomas donde se decidió seguimiento evolutivo. En la actualidad sigue libre de síntomas y los estudios de imagen no muestran datos de recidiva local o metástasis a distancia a los 18 meses de la cirugía.

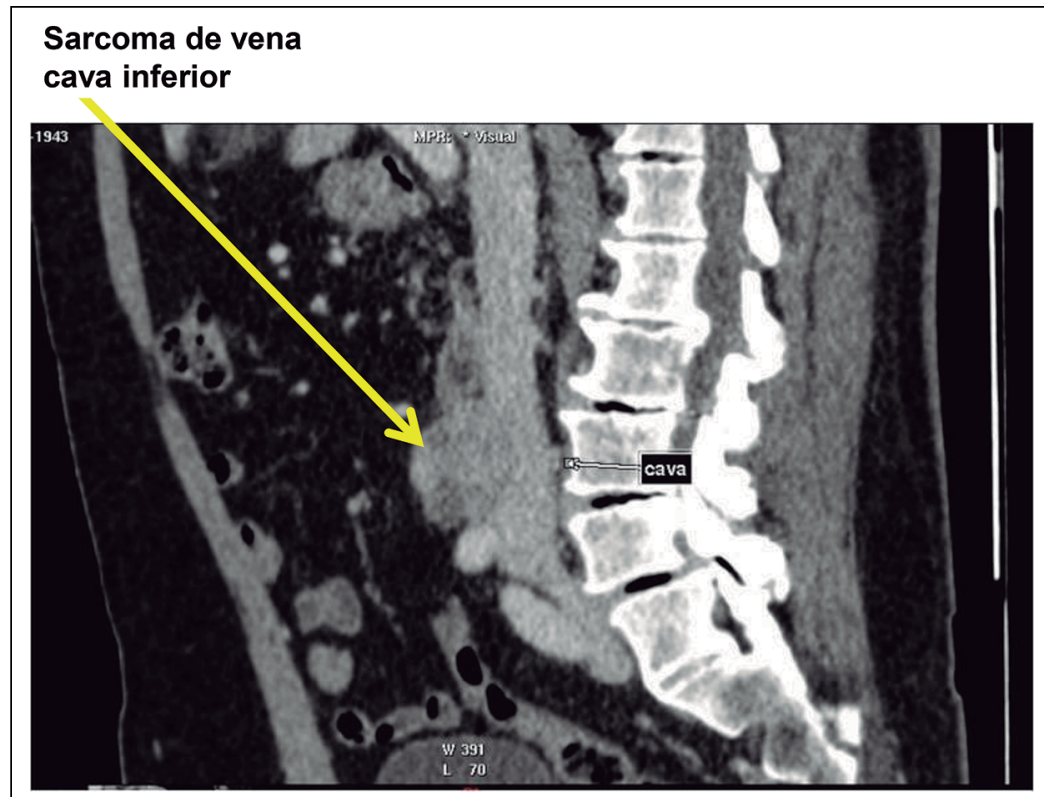

Figura 1. Corte sagital de TC donde se observa la masa retroperitoneal que corresponde a leiomiosarcoma de $63 \times 52 \mathrm{~cm}$ de la vena cava inferior.

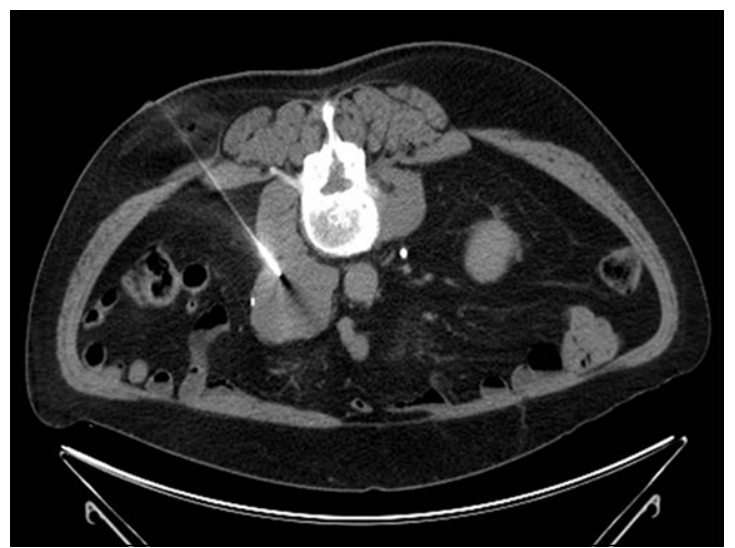

Figura 2. BAG guiada por tomografía computada. 


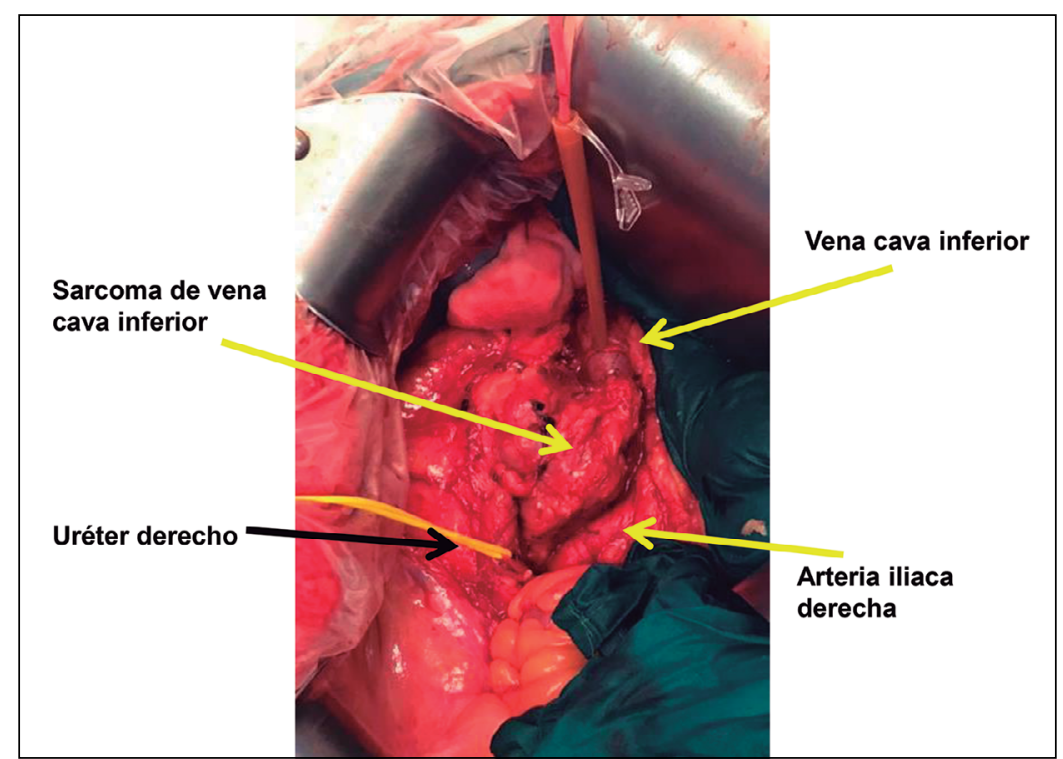

Figura 3. Campo quirúrgico donde se observa el leimiosarcoma de cava, la vena cava controlada con un torniquete de Rummel, el uréter derecho y la arteria iliaca común derecha.

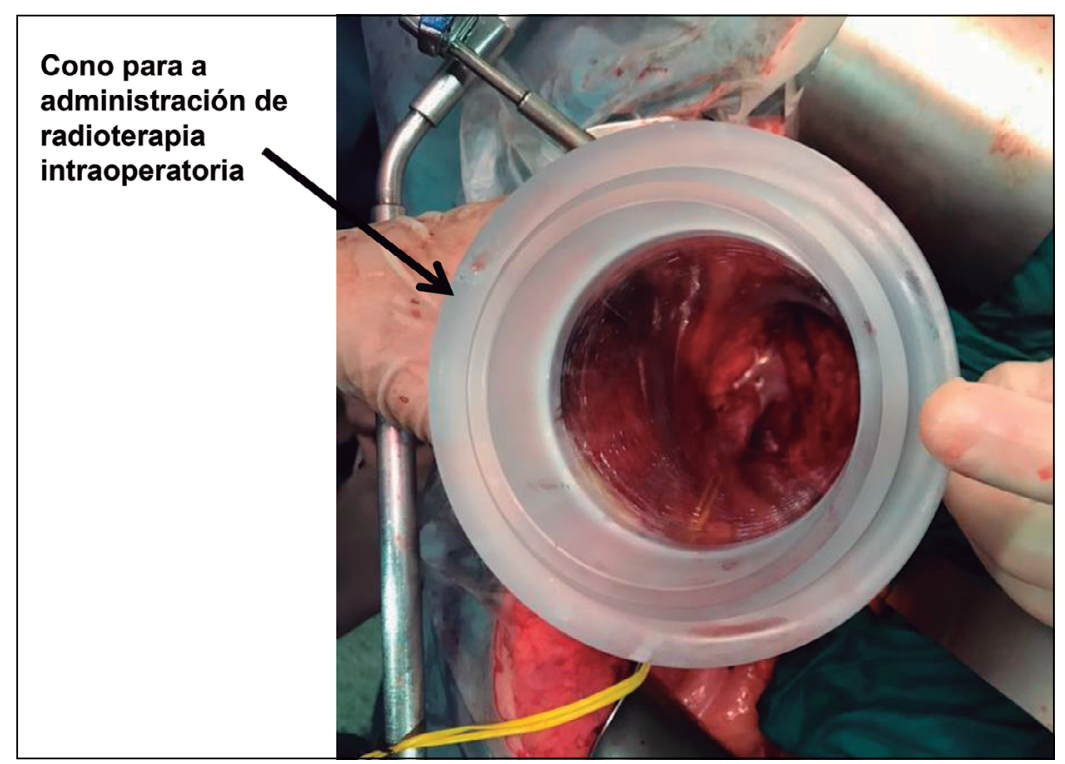

Figura 4. Cono para la aplicación de la radioterapia intraoperatoria en el lecho quirúrgico.

\section{Discusión}

El leiomiosarcoma de VCI representa el $0,5 \%$ de todos de los sarcomas y el $15 \%$ de los tumores retroperitoneales ${ }^{3,4}$. En nuestro caso se trataba de un varón que es algo poco habitual en este tipo de tumores.

La clínica suele ser inespecífica y en más del
$10 \%$ de los casos se trata de un hallazgo casual asintomático, como ocurrió en nuestro paciente. Los síntomas descritos con más frecuencia son el dolor abdominal inespecífico, masa abdominal palpable y edema en los miembros inferiores. Otros síntomas asociados son pérdida de peso, síndrome de BuddChiari o síndrome de vena cava inferior ${ }^{1}$.

El diagnóstico requiere de la realización de pruebas de imagen que aportan información sobre la localización exacta del tumor y su relación con estructuras vecinas muy importante para planificar la cirugía, pero sólo con dichas pruebas puede ser difícil diferenciar entre sarcoma retroperitoneal que afecta a la VCI o leimiosarcoma de $\mathrm{VCI}^{6}$. Por lo que es necesaria la confirmación histológica que precisa de la realización de una biopsia con aguja gruesa (BAG), que en nuestro paciente es lo que estableció el diagnóstico definitivo de leiomiosarcoma de vena cava.

La clasificación más empleada descrita por Kulayat es por su localización, en la que se divide la VCI en tres segmentos o niveles:

Segmento o nivel 1: distal a las venas renales (36\% de los casos).

Segmento o nivel 2: entre las venas renales y las venas suprahepáticas, constituyen el grupo de mejor pronóstico (44\% de los casos).

Segmento o nivel 3: desde la desembocadura de la aurícula derecha hasta las venas suprahepáticas $(20 \% \text { de los casos })^{2-4,7}$.

El tratamiento quirúrgico con resección completa de la lesión es el único que parece influir en la supervivencia a largo plazo $^{2-4}$. En los de nivel I como nuestro caso, se debe realizar resección total del tumor y de la porción de la VCI comprometida. Tras la resección puede realizarse ligadura de la VCI infrarrenal o colocación de prótesis biológicas (pericardio bovino) o sintéticas (prótesis anilladas de polietrafluoroetileno). Actualmente, no hay evidencia suficiente que revele cuál es la mejor opción porque ninguna está exenta de riesgos. La ligadura aumenta el riesgo de TVP como ocurrió en nuestro paciente aunque sin posibilidad de tromboembolismo pulmonar (TEP), mientras que la reconstrucción con prótesis suele trombosarse con el elevado riesgo de TEP o infectarse. No hay consenso en la necesidad de anticoagulación o antiagregación tras la cirugía ${ }^{3}$. En el caso de nuestro paciente recibió profilaxis tromboembólica con heparina de bajo peso y tras establecerse la TVP se administraron dosis anticoagulantes.

En la actualidad, el papel de la adyuvancia y neoadyuvancia en estos tumores es muy controvertido $^{3,4,7}$. Las guías clínicas de la NCCN publican 
que no existe evidencia que la quimioterapia influya en la supervivencia de los pacientes ${ }^{7,9}$. También refieren que la quimioterapia preoperatoria es una alternativa aceptable en tumores no resecables con una recomendación $2 \mathrm{~B}$ y si tras la quimioterapia se produce regresión del tumor se recomienda la cirugía. La RT preoperatoria parece tener un papel en la disminución del tamaño tumoral que facilita la cirugía, en nuestro caso creó un edema peritumoral que facilitó la disección del tumor. Sin embargo, no hay estudios randomizados que hayan demostrado su eficacia en aumentar la supervivencia. Las guías NCCN le dan una recomendación $2 \mathrm{~B}^{3,7,9}$. En cuanto a la RIO un estudio prospectivo que compara la RT externa (pre o post) y RIO con RT externa no demostró ninguna diferencia significativa con respecto al tiempo de supervivencia libre de enfermedad, aunque sí se observó una disminución de la incidencia de enteritis rádica ${ }^{10}$.

El factor más importante para aumentar la supervivencia es obtener unos márgenes libres de tumor. La tasa de supervivencia a 2 y 5 años en pacientes con márgenes de resección libres es del 90 y $66 \%$, respectivamente. Cuando los márgenes son positivos es de 21 meses, y en aquellos en los que no se realizó una resección completa del tumor, la supervivencia disminuye a 8 meses. Los pacientes con tumores no resecables tienen una supervivencia de pocos meses y las resecciones paliativas pueden aliviar los síntomas. No se ha conseguido correlacionar el tamaño, la edad, ni la presencia de ganglios positivos con la supervivencia ${ }^{3}$.

\section{Conclusiones}

Debido a la escasa frecuencia de estos tumores, los mejores resultados se obtienen con un abordaje multidisciplinar por parte de equipos experimentados en centros de referencia.

\section{Responsabilidades éticas}

Protección de personas y animales. Los autores declaran que para esta investigación no se han realizado experimentos en seres humanos ni en animales.

Confidencialidad de los datos. Los autores declaran que en este artículo no aparecen datos de pacientes.

Conflicto de Intereses: Ninguno.

\section{Referencias}

1. Kim JT, Kwon T, Cho Y, Shin S, Lee S, Moon D. Multidisciplinary treatment and long-term outcomes in six patients with leiomyosarcoma of the inferior vena cava. J Korean Surg Soc. 2012;82:101-9.

2. Centeno M, Zaldívar Reyna O, Abohar, Medina C, Padrón Rivera L. Leiomiosarcoma retroperitoneo primario de vena cava. Reporte de un caso y revisión de la literatura. Rev Hosp Jua Mex. 2011;78:46-9.

3. López-Ruiz JA, Tallón-Aguilar L, Marenco-de la Cuadra B, López-Pérez J, Oliva-Mompeán F, Padillo-Ruiz J. Leiomyosarcoma of the inferior vena cava. Case report and literature review.
Cirugía y Cirujanos (English Edition) 2017;85:361-5.

4. García M, Messa O, Ríos D, López H, Canedo J. Leiomiosarcoma de vena cava inferior: reporte de un caso y revisión de la literatura. Rev Colomb Cancerol. 2014;18:41- 5.

5. Jáuregui-Francia FT, Jáuregui-Caycho L, Jauregui-Figueroa MR, Pirilla-Janto JM. Leiomiosarcoma de vena cava, reporte de un caso. Rev Fac Med Hum. 2017;17:947.

6. Nascif RL, Santana AG, Lacerda G Caldas G, de Araujo V, Canuto MR. Radiol Bras. 2014;47:384-6.

7. Spinelli A, Schumacher G, Benckert C, Sauer IM, Schmeding M, Glanemann $\mathrm{M}$, et al. Surgical treatment of a leiomyosarcoma of the inferior vena cava involving the hepatic and renal veins confluences: Technical aspects. EJSO 2008;34:831-5.

8. Kieffer E, Alaoui M, Piette JC, Cacoub P, Chiche L. Leiomyosarcoma of the inferior vena cava: experience in 22 cases. Ann Surg. 2006;244:289-95.

9. Zarebczan B, Smith B, Tefera G, Weber S. Surgical management of retroperitoneal leiomyosarcoma arising from the inferior vena cava. J Gastrointest Surg. 2013;17:2166-71.

10. García Sabrido JL, Velasco E, Calvo F, Gómez Lanz L, Sánchez Tocino J, Argudo Garijo S, et al. Intensificación terapéutica intraoperatoria en el tratamiento de los sarcomas abdominales localmente avanzados. Cir Esp. 2006;80:200-5. 